\title{
EFFECTIVENESS OF ULTRASOUND-GUIDED CORTICOSTEROID INJECTION AND ALCOHOL NEUROLYSIS IN THE TREATMENT OF MERALGIA PARESTHETICA DURING A 28-MONTH FOLLOW-UP PERIOD: A RETROSPECTIVE COHORT STUDY
}

\author{
Özge Yapıc1 ${ }^{1}$ and Meriç Uğurlar ${ }^{2}$ \\ ${ }^{1}$ Marmara University School of Medicine \\ ${ }^{2}$ Silivri Anadolu Private Hospital
}

June 2, 2021

\begin{abstract}
Abstract Objectives Meralgia paresthetica is a very rare sensory mononeuropathy of the lateral femoral cutaneous nerve (LFCN). The purpose of this study was to evaluate the outcomes and compare the results of ultrasound-guided corticosteroid injection and ultrasound-guided alcohol neurolysis in the treatment of meralgia paresthetica. Methods We performed a retrospective clinical study of 26 patients with a diagnosis of marelgia paresthetica with a duration of [?]10 months. The patients were divided into 2 groups, with the Group 1 receiving ultrasound-guided local corticosteroid injection and Group 2 receiving ultrasoundguided alcohol neurolysis to the entrapment site of the LFCN. Results The mean age of the patients in Group 1 was 42.2 years and in Group 2 was 40.8 years. The mean follow-up period of Group 1 was 28.7 months and Group 2 was 28.4 months. At the end of the follow-up period 9 patients in Group 1 and 10 patients in Group 2 declared full pain relief and improvement in cutaneous sensitivity. Conclusion Once meralgia paresthetica has persisted corticosteroid injection and alcohol neurolysis are both effective methods. Although the recurrence rates are higher in corticosteroid injection, both treatment methods decreased the pain and improved the patients' satisfaction and long-term curative effect.
\end{abstract}

EFFECTIVENESS OF ULTRASOUND-GUIDED CORTICOSTEROID INJECTION AND ALCOHOL NEUROLYSIS IN THE TREATMENT OF MERALGIA PARESTHETICA DURING A 28-MONTH FOLLOW-UP PERIOD: A RETROSPECTIVE COHORT STUDY

\section{Abstract \\ Objectives}

Meralgia paresthetica is a very rare sensory mononeuropathy of the lateral femoral cutaneous nerve (LFCN). The purpose of this study was to evaluate the outcomes and compare the results of ultrasound-guided corticosteroid injection and ultrasound-guided alcohol neurolysis in the treatment of meralgia paresthetica.

\section{Methods}

We performed a retrospective clinical study of 26 patients with a diagnosis of marelgia paresthetica with a duration of [?]10 months. The patients were divided into 2 groups, with the Group 1 receiving ultrasound-guided local corticosteroid injection and Group 2 receiving ultrasound-guided alcohol neurolysis to the entrapment site of the LFCN. 


\section{Results}

The mean age of the patients in Group 1 was 42.2 years and in Group 2 was 40.8 years. The mean follow-up period of Group 1 was 28.7 months and Group 2 was 28.4 months. At the end of the follow-up period 9 patients in Group 1 and 10 patients in Group 2 declared full pain relief and improvement in cutaneous sensitivity.

\section{Conclusion}

Once meralgia paresthetica has persisted corticosteroid injection and alcohol neurolysis are both effective methods. Although the recurrence rates are higher in corticosteroid injection, both treatment methods decreased the pain and improved the patients' satisfaction and long-term curative effect.

Key words: Meralgia paresthetica; nerve entrapment; ultrasound; alcohol neurolysis; corticosteroid.

\section{INTRODUCTION}

Meralgia paresthetica is a well-known but very rare sensory mononeuropathy of the lateral femoral cutaneous nerve (LFCN) resulting from an entrapment of the LFCN. ${ }^{1,2}$ Meralgia paresthetica was first described by Hager in $1885 .{ }^{3}$ In 1895 , Bernhardt reported more extensive report about this neuropathy and two weeks later Roth published an article emphasizing the term meralgia that comes from the Greek words "meros" for thigh and "algos" for pain. ${ }^{3}$ This condition is also known as "Bernhardt-Roth syndrome". ${ }^{4}$ Classically meralgia paresthetica results with dysesthesia or anesthesia in the anterolateral thigh in the distribution of the LFCN. ${ }^{3}$ The ligtning pain, burning, coldness, numbness, tingling, muscle achiness, frank anesthesia or even local hair loss in the anterolateral thigh is typically described by the patients. ${ }^{1}$

Generally patients get pain relief by conservative treatment methods. ${ }^{3}$ However, patients who do not get benefit from conservative treatment local corticosteroid injections, alcohol neurolysis, pulsed radiofrequency or even surgical decompression may be required., ${ }^{1,5-7}$

The purpose of this study was to evaluate the outcomes and compare the results of ultrasound-guided corticosteroid injection and ultrasound-guided alcohol neurolysis in the treatment of meralgia paresthetica. To the best of our knowledge, this is the first study in the English literature comparing these two treatment modalities in the treatment of meralgia paresthetica.

\section{PATIENTS AND METHODS}

\subsection{Study desing and participants}

We performed a retrospective clinical study of 26 patients with a diagnosis of marelgia paresthetica with a duration of [?]10 months from August 2012 and April 2017. The patients were divided into 2 groups, with the Group 1 receiving ultrasound-guided local corticosteroid injection and Group 2 receiving ultrasound-guided alcohol neurolysis to the entrapment site of the LFCN. The study was designed according to the STROBE checklist for observational studies and approved by the Institutional Review Board.

\subsection{Clinical diagnosis and clinical follow-up}

The diagnosis of all the patients in both groups were established by clinical history, physical examination, electromyographic findings (lateral cutaneous nerve amplitude potential $<10 \mu \mathrm{V}$, latency $>3.5$ milliseconds, and normal thigh muscle needle examination findings in electromyography (EMG) ${ }^{8,9}$ In physical examination all the patients had disquieting severe pain over the anterolateral thigh in the distribution of LFCN with some sensory loss. According to the anamnesis of the patients activities such as walking, climbing stairs, and hip extension aggravated the pain. There was a clear sensory loss of all patients in the sensory examination in the distribution of LFCN. All the patients had normal motor examination. All cases in both groups were evaluated according to the inclusion and exclusion criteria (Table 1 and 2). All the patients in both groups had unilateral symptoms. 
All the patients were confirmed by electrodiagnostic studies showing attenuated sensory nerve action potential (SNAP) amplitudes or reduced sensory nerve conduction velocity (SNCV). ${ }^{10,11}$ Spinal magnetic resonance imaging (MRI) was performed to all the patients and there was no sign of lumbar disease in the MRI of all the patients.

All patients in both groups referred to our clinic due to a failure of at least two of the conservative treatment methods, such as nonsteroidal anti-inflammatory drugs (NSAIDs), physical therapy, pregabaline, gabapentin, amitriptyline, looser clothing, and weight loss for 6 months. The patients who did not experience benefit from these conservative methods at the end of the 6-month follow-up period were indicated for injection. All patients were informed about the procedure and objectives of the injections as well as possible complications, and they have signed an informed consent form before the injections.

The pre-treatment assessments in both groups included a complete history, physical examination, and laboratory tests, including complete blood cell and platelet counts, erythrocyte sedimentation rate, C-reactive protein level, prothrombin time, partial thromboplastin time, blood urea nitrogen, creatinine level, and electrolyte level analysis.

The pre-treatment and post-treatment collected data in both groups included the patients' self-assessments of thigh pain, activity and function level, use of analgesics, EMG evaluation findings, adverse events, and complication data.

The patients who were allocated to the study were advised to avoid using any other conservative treatment, such as NSAIDs, physical therapy, pregabaline, gabapentin, amitriptyline. Of the 26 patients, 15 were assigned to the Group 1 and 11 to the Group 2. The study population included 11 male and 4 female cases in Group 1 and 10 male and 1 female in Group 2. The demographic data of the subjects are summarized in Table 3. The severity of pain, before and after injections, during the last 24 hours at rest and during daily activities at the distribution of LCFN was recorded using a visual analog scale (VAS) score, ranging from 0 to 10 , with 0 indicating no pain and 10 indicating severe pain. The degree of numbness, before and after injections, (none; slight; moderate; and severe) was recorded. ${ }^{12}$

The patients in both groups received 3 injections once each month with an interval of 30 days between the sessions. All the injections were performed using real-time ultrasonography (USG) guidance with a linear array transducer. The USG evaluation was performed by visualization of the LCFN of the affected thigh and nonaffected thigh for comparison before and after the injections. All USG examinations of the patients' symptomatic LFCN and injection procedures were performed by the same radiologist as described by Tagliafico et al. ${ }^{13}$ All the patients in both groups were advised to apply local ice to their thigh and to take $500 \mathrm{mg}$ paracetamol tablets 3 times daily to relieve pain for 3 days in the post-injection period. No activity or weight-bearing limitation was advised to the patients.

In the last follow-up we drew-up a simple survey for all patients and we asked the patients whether they were satisfied with the outcome of the injection and if they feel the same pain, do they get the same injection again. The results of this survey was analyzed according to the etiology of marelgia paresthetica, the duration of the symptoms, the excess weight of the patients, and the electrodiagnostic findings. The assessment of the statistical analysis was done by chi-square test.

\subsection{Ultrasound technique}

The described technical USG approach was adapted from the previous sudies. ${ }^{8,13,14}$ All USG examinations and injections of the patients' were performed by the same radiologist. With the patient in supine position, the anterior superior iliac spine was palpated as a landmark, and was visualized by a ultrasound transducer as a hypoechoic structure with posterior acoustic shadowing. The lateral side of the ultrasound transducer was placed on the anterior superior iliac spine. Then the medial side of the ultrasound transducer extended medially in an anatomic transverse plane. When the ultrasound transducer was in this position, the medial side of the ultrasound transducer was slightly angled in a caudal direction. According to Tagliafico et al., at this position the transducer was parallel to the inguinal ligament. ${ }^{15}$ Then the radiologist moved 
the ultrasound transducer gently in a mediocaudal direction until the echo signature of the LFCN was determined. LFCN was seen as a typical hypoechoic oval structure. USG can show hypoechoic swelling, cross-sectional area enlargement, and perineural fibrosis in the entrapment site. ${ }^{14}$

\subsection{Injection technique}

Under USG guidance, in Group 1, $2 \mathrm{~mL}$ of bupivacaine $5 \mathrm{mg} / \mathrm{mL}$ and after 3 minutes $1 \mathrm{~mL}$ of betamethasone $40 \mathrm{mg} / \mathrm{mL}$, and in Group 2, $2 \mathrm{~mL}$ of bupivacaine $5 \mathrm{mg} / \mathrm{mL}$ and after 3 minutes $3 \mathrm{~mL}$ of $75 \%$ ethanol were injected into the site of maximal tenderness, around the LCFN at the level where USG showed pathological alterations of the nerve calibre, by using a $23 \mathrm{G}$ needle in sterile conditions (appropriate skin preparation and disinfection, sterile USG gel and transducer, and patient sterile covering). ${ }^{14}$

\subsection{Statistical analysis}

A sample size of 10 patient per group was required to provide $80 \%$ power to detect differences at an $\alpha$ level of 0.05 to indicate significance. The results are presented as the means \pm standard deviation (SD), number, and percentage. A normality test was performed before the statistical analysis with t-test. The comparisons were performed using the chi-square test or Fisher's exact test for count data (gender, ASA grade, and treatment level), and the independent-sample t-test for measurement data (age, body mass index). The change in VAS was analysed by Two-way analysis of variance. The degree of numbness and the satisfaction of patients were analysed by repeated-measures analysis of variance. $p<0.05$ was considered statistically significant. SPSS 20.0 was used to perform statistical analysis.

\section{RESULTS}

\subsection{Demographics and patient clinical features}

None of the patients in both groups were lost to follow-up. The groups were similar to each other at baseline demographics such as gender, age, American Society of Anesthesiologists (ASA) grade, and duration of symptoms (Table 3). The mean age of the patients in Group 1 was $42.2 \pm 8.4$ (range: 27-55) years and in Group 2 was $40.8 \pm 7.3$ (range: 31-52) years. The mean follow-up period of Group 1 was $28.7 \pm 2.6$ months (range: 24-33) and Group 2 was $28.4 \pm 2.7$ months (range: 24-34). The mean body mass index (BMI) in Group 1 was $29 \pm 2.5 \mathrm{~kg} / \mathrm{m}^{2}$ (range: $24.3-32.3$ ) and in Group 2 was $30 \pm 3.4 \mathrm{~kg} / \mathrm{m}^{2}$ (range: 22.9-34.3). The mean interval between the onset of pain and admission to the hospital of the patients was $12.4 \pm 0.9$ (range: 11-14) months in Group 1 and $12.1 \pm 1.2$ (range: 10-14) in Group 2. The pre-injection mean VAS score in Group 1 was $7.6 \pm 1.05$ (range: 6-9), and in Group 2 was $7.5 \pm 1.03$ (range: 6-9). There were no significant difference between the gender, age, BMI, ASA scores, duration of symptoms, mean VAS scores, and follow-up period between the two groups.

At the end of the follow-up period 9 patients in Group 1 and 10 patients in Group 2 declared full pain relief and improvement in cutaneous sensitivity and they were completely satisfied. At the end of the follow-up period 2 patients in Group 1 and 1 patient in Group 2 declared partial recovery in pain and partial improvement in cutaneous sensitivity but they were also satisfied with the injection. At the end of the follow-up period 4 patients in Group 1 had partial or full improvement in pain and in cutaneous sensitivity in the post-injection first year but at the end of the follow-up period they had recurrence of the symptoms. None of the patients in Group 2 had recurrence of the symptoms at the end of the follow-up period. The mean period until pain relief and improvement in cutaneous sensitivity was $14.8 \pm 1.8$ months (range: 12-19) in Group 1 and $14.5 \pm$ 2.0 months (range: 12-18) in Group 2. At the end of the follow-up period the post-injection mean VAS score in Group 1 was $2.9 \pm 2.3$ (range: 1-7), and in Group 2 was $1.8 \pm 0.7$ (range: 1-3). The differences between the post-injection mean VAS scores of the two groups at the end of the follow-up period was due to the recurrence of the symptoms of 4 patients in Group 1. All the patients in both groups reported that the degree of numbness was aggravated with time in the post-injection first week till the post-injection first month, and the degree of numbness at post-injection first week and first month in Group 2 was greater than the Group $1(p<0.05)$. However, none of the patients in both groups complained about the post-injection aggravation in the degree of numbness. Although the numbness was resolved in all patients in Group 2 at the end of the 
follow-up period, 4 patients in Group 1 reported the recurrence of numbness from the post-injection first year till the end of the follow-up period. The satisfaction ratio of patients in both groups at 1-week and 1-month after the injection did not significantly differ $(p<0.05)$. However, the satisfaction ratio in Group 2 was greater than Group 1 at the end of the follow-up period $(p<0.05)$.

\subsection{Complications}

There were some complications after the injections. Three patients in Group 1 had discloration in the skin after the corticosteroid injection and 1 patient in Group 2 had local hematoma without requiring drainage at the injection site. The patient with hematoma was treated with $445 \mathrm{mg}$ mucopolysaccharide polysulfate (chondroitin polysulfate) cream applied over the skin. The discoloration in the skin of 3 patients in Group 1 was recovered in the last follow-up period. The alcohol neurolysis and corticosteroid injection induced severe pain (flare reaction) when injected in 3 patients in Group 1 and in 3 patients in Group 2. The aggravation of the pain affected the cooperation and satisfaction of the all patients. However, the aggravation of the pain in these patients resolved at the post-injection second day and all these patients were satisfied with the injection at the end of the follow-up period. There were no other complications, such as infection, and local anesthetic toxicity were recorded.

\section{DISCUSSION}

Meralgia paresthetica is usually idiopathic but it can ocur due to trauma, overuse, obesity, pregnancy, tight fitting clothing, wearing heavily loaded hip belts, pelvic and retroperitoneal tumors, streching of the nerve due to prolonged leg hyperextension, prolonged standing, leg length discrepancies, scoliosis, periostitis of the ilium, lower abdominal and pelvic incisions (Pfannenstiel incisions and appendectomies), laparoscopic hernia repairs, Chiari pelvic osteotomies, and iliac bone graft harvesting. $3,16,17$

The diagnosis of meralgia paresthetica is based on the clinical findings and the diagnosis can be confirmed by nerve conduction studies showing decrease in the amplitude of SNAP with side-to-side amplitude difference. ${ }^{1,11}$ In some cases the diagnosis can be confirmed by the LCFN block with local anaesthetic. ${ }^{18}$ In our patients, there were both of the positive nerve conduction study and positive blocks with local anaesthetic in both groups.

Meralgia paresthetica can be seen in any age group, especially between the ages of 30 and $65 .^{3}$ Although most authors reported a male predominance, ${ }^{3,19,20}$ only Rosencheck reported an equal distribution between the genders. ${ }^{21}$ Whereas, there are some authors reported a female predominance. ${ }^{3,22}$ In present study, the age ranges of the patients were between 27 and 55 with a male predominance.

There are no concensus on the treatment of meralgia paresthetica and no clear protocols or guidelines are reported. ${ }^{23}$ Nonoperative treatment, such as NSAIDs, heat, and physical therapy is usually sucessful. ${ }^{24}$ Pregabaline, gabapentin, and amitriptyline have been tried for the treatment, although these drugs have significant adverse effects. ${ }^{16,23}$ Local anesthetic agent injections with or without perineural corticosteriod such as triamcinolone, dexamethasone, betamethasone or methylprednisolone have been shown to be effective in case series. ${ }^{7,23,25,26}$ In a prospective study there is some evidence of perineural corticosteroid injections, whereas alcohol neurolysis has a limited evidence. ${ }^{1,5,13}$ A Cochrane meta-analysis of observational studies reported that because of the absence of any published randomised controlled trials (RCTs) or quasi-RCTs the effect of local corticosteroid injections is weak. ${ }^{27}$

Although many kinds of neurolytic agents are used in the clinics, alcohol is the most widely used agent. ${ }^{28}$ Dehydrated alcohol is colorless and transparent, and the concentration is greater than $99.5 \%$ when used. ${ }^{28}$ The alcohol provides pain relief in patients by contacting the nerves directly, destroying the nerve structure, and blocking nerve conduction. ${ }^{29}$

Alcohol neurolysis of the LFCN has been effective to treat meralgia paresthetica. ${ }^{5}$ However, there have been isolated reports of alcohol neurolysis being a safe and effective method in the treatment of meralgia paresthetica, no large study has evaluated the use of alcohol neurolysis in this context. ${ }^{5,30}$ Chen et al. 
provided pain relief for recurrent meralgia paresthetica with alcohol neurolysis. ${ }^{5}$ Hung et al. reported longterm control of malignant abdominal wall pain with alcohol neurolysis of transversus abdominal plane block. ${ }^{31}$ Fujita decreased upper abdominal cancer pain of the patients with splanchnic nerve alcohol neurolysis. ${ }^{32}$ In this study, all patients had pain relief with alcohol neurolysis as indicated by the reduced VAS scores. This meant that alcohol was effective in the treatment of marelgia paresthetica.

In this retrospective study, LFCN corticosteroid injection and alcohol neurolysis was successfully implemented in all patients. The results showed that the alcohol neurolysis decreased the VAS scores in all patients at the end of the follow-up period and when compared with the corticosteroid injection alcohol neurolysis much more improved the satisfaction of patients. Haim et al. retrospective study of 79 patients. Twenty-one patients were treated by conservative treatment. The remaining 58 patients who did not have any benefit from the conservative treatment were treated by betamethasone injection. Complete relief occured in 22 patients after the first injection, in 12 patients after the second injection, and in 14 patients after the third injection. However, 10 patients did not have any benefit from the corticosteroid injections. ${ }^{33}$ Ivins and Tumber et al. reported pain relief in $50 \%$ of their patients after the local anesthetic and methylprednisolone injection. ${ }^{3,34}$ Elavarasi et al. reported a cohort study of 8 patients treated with triamcinolone injections. After the 16 months of follow-up $75 \%$ patients had complete pain relief and all patients had $50 \%$ reduction in symptoms. ${ }^{23}$

The curative effect of alcohol can be increased by increasing the alcohol concentration. ${ }^{35}$ By increase in the alcohol concentration increases the nerve injury degree. ${ }^{35}$ In a study, it was emphasized that $99.9 \%$ alcohol was more effective than the $50 \%$ and $75 \%$ alcohol on the rat sciatic nerves. ${ }^{35}$ We injected $3 \mathrm{~mL}$ of $75 \%$ ethanol to our patients and this amount of alcohol was found effective.

Alcohol injection can induce temporary severe pain due to local irritation. ${ }^{35,36}$ There are two causes of this effect. First, when the alcohol and local anesthetic agent are both diluted for the injection to the lesion site, the analgesic effect of the local anesthetic decreases. Second, when the local anesthetic is injected previously, the diffusion range of alcohol, which is injected as the anesthesia, may be widened. ${ }^{35}$ Hung et al. got longer pain relief with high concentration of alcohol in neurolytic transversus abdominal plane block than the low concentration of alcohol. ${ }^{31}$

A wide range of incidences of flare reaction $1 \%$ to $81 \%$ due to corticosteroid injection at different anatomical locations have been reported by various studies. ${ }^{37,38}$ Hollander has treated 100.000 patients with corticosteroid injections and reported post-injection inflammatory flare incidence in approximately $1 \%$ to $2 \%$. Hollander reported that the flare reaction was related with larger doses of corticosteroid and traumatic injections. ${ }^{38}$ The flare reaction after corticosteroid injection was studied in detail by McCarty and Hogan. They determined that although the corticosteroid injection has an anti-inflammatory effect, the corticosteroid crystal acts as a local irritant. ${ }^{39}$ The flare reaction due to corticosteroid injection usually starts a few hours later after the injection and resolves spontaneously within 72 hours. ${ }^{40}$ In our study, the flare reaction in all patients in both groups resolved in approximately post-injection 24 hours. The post-injection pain is not because of the inadequate local anesthesia. As in our patients, the LCFN block before the injections at the lesion site can provide some pain relief in the post-injection period and this can improve patients' satisfaction. Kim et al. and Akural et al. get partially pain relief in their patients in the post-injection period of the nerve neurolysis. ${ }^{41,42}$ There was flare reaction in both of our groups and the aggravation of the pain in these patients resolved at the post-injection second day.

\subsection{Limitations}

To the best of our knowledge, ours is the first study designed to compare the use of corticosteroid injection and alcohol neurolysis for the treatment of meralgia paresthetica. However, the present study did have some limitations. First, we had a small number of patients in both groups, which resulted from the inclusion and exclusion criteria for our study. The results needed further support by a larger number of patients. Second, we did not include a placebo control group in our study. Additionally, the ultrasound outcomes were not evaluated by using a blinded method. Our study is a retrospective study. However, a prospective, randomized, 
placebo-controlled, double-blind study would be an ideal study. Nonetheless, the primary aim of our study was to compare the two treatment methods, not to show their individual effects. In addition, we did not assess the changes of the LFCN lesions using pre-treatment and post-treatment ultrasound imaging or MRI. The distribution of injected corticosteroid or alcohol was not clear because a contrast agent was not used. Although limited by many factors, the results of our series have shown that there was improvement between the pre-injection and post-injection mean VAS scores for both groups at the end of the follow-up period. However, the improvement in mean VAS scores was better in Group 2. The reason of this was due to the recurrence of the symptoms of 4 patients in Group 1. The increase in activity level of the patients, changes in lifestyle, weight gain, and changes in clothes could have affected the results. Thus, another important limitation of our study was the difficulty in controlling for all these changes in 26 patients at the mean 28month follow-up period. Therefore, more prospective, randomized, placebo-controlled, double-blind studies are needed. Finally, a longer follow-up period might reveal more benefits and handicaps of these treatment methods. The strengths of our study included the mean 28-month comprehensive assessment of the outcomes. The present study provides valuable information for future treatment trials of meralgia paresthetica.

\section{CONCLUSION}

In conclusion, once meralgia paresthetica has persisted corticosteroid injection and alcohol neurolysis are both effective methods. Although the recurrence rates are higher in corticosteroid injection, both treatment methods decreased the pain and improved the patients' satisfaction and long-term curative effect.

\section{ACKNOWLEDGEMENTS}

None.

\section{CONFLICT OF INTEREST DISCLOSURE}

All named authors hereby declare that they have no conflicts of interest to disclose. This research received no specific grant from any funding agency in the public, commercial, or not-for-profit sectors.

\section{AUTHOR CONTRIBUTIONS}

The manuscript has been read and approved by all the authors, that it has not been submitted to, or is not under consideration for publication in another journal. All authors were involved in all parts of study and manuscript preparation including literatüre research, study design, analysis of data, manuscript preparation, and review of the manuscript.

\section{DATA AVAILABILTY STATEMENT}

Data available on request from the authors.

\section{ETHICAL STATEMENT}

The study was approved by our institutional review board and ethics committee. The authors are accountable for all aspects of the work in ensuring that questions related to the accuracy or integrity of any part of the work are appropriately investigated and resolved.

\section{REFERENCES}

1. Ahmed A, Arora D, Kochhar AK. Ultrasound-guided alcohol neurolysis of lateral femoral cutaneous nerve for intractable meralgia paresthetica: a case series. Br J Pain. 2016;10(4):232-237.

2. Dharmasaroja P, Dharmasaroja P. Meralgia paresthetica-like syndrome may be caused by transient lumbar nerve root injury without definite compression: a case report. J Med Assoc Thai.2010;93(Suppl.7):S307-310.

3. Ivins GK. Meralgia paresthetica, the elusive diagnosis: clinical experience with 14 adult patients. Ann Surg.2000;232(2):281-286.

4. Kallgren MA, Tingle LJ. Meralgia paresthetica mimicking lumbar radiculopathy. Anesth Analg. 1993;76(6):1367-1368. 
5. Chen CK, Phui VE, Saman MA. Alcohol neurolysis of lateral femoral cutaneous nerve for recurrent meralgia paresthetica. Agri.2012;24(1):42-44.

6. Ghai B, Dhiman D, Loganathan S. Extended duration pulsed radiofrequency for the management of refractory meralgia paresthetica: a series of five cases. Korean J Pain. 2018;31(3):215-220.

7. Harney D, Patijn J. Meralgia paresthetica: diagnosis and management strategies. Pain Med. 2007;8(8):669-677.

8. Bodner G, Bernathova M, Galiano K, Putz D, Martinoli C, Felfernig M. Ultrasound of the lateral femoral cutaneous nerve: normal findings in a cadaver and in volunteers. Reg Anesth Pain Med.2009;34(3):265-268.

9. Kalichman L, Vered E, Volchek L. Relieving symptoms of meralgia paresthetica using Kinesio taping: a pilot study. Arch Phys Med Rehabil. 2010;91(7):1137-1139.

10. Lagueny A, Deliac MM, Deliac P, Durandeau A. Diagnostic and prognostic value of electrophysiologic tests in meralgia paresthetica. Muscle Nerve. 1991;14(1):51-56.

11. Seror P, Seror R. Meralgia paresthetica: clinical and electrophysiological diagnosis in 120 cases. Muscle Nerve.2006;33(5):650-654.

12. Zhao WX, Wang Q, He MW, Yang LQ, Wu BS, Ni JX. Radiofrequency thermocoagulation combined with pulsed radiofrequency helps relieve postoperative complications of trigeminal neuralgia. Genet Mol Res. 2015;14(3):7616-7623.

13. Tagliafico A, Serafini G, Lacelli F, Perrone N, Valsania V, Martinoli C. Ultrasound-guided treatment of meralgia paresthetica (lateral femoral cutaneous neuropathy): technical description and results of treatment in 20 consecutive patients. J Ultrasound Med.2011;30(10):1341-1346.

14. Klauser AS, Abd Ellah MM, Halpern EJ, Sporer I, Martinoli C, Tagliafico A, Sojer M, Taljanovic MS, Jaschke WR. Meralgia paraesthetica: Ultrasound-guided injection at multiple levels with 12-month follow-up. Eur Radiol. 2016;26(3):764-770.

15. Tagliafico A, Padua L, Martinoli C. High-resolution ultrasonography in the assessment of meralgia paresthetica: some clarifications are needed. Muscle Nerve. 2012;45(6):922.

16. Mulvaney SW. Ultrasound-guided percutaneous neuroplasty of the lateral femoral cutaneous nerve for the treatment of meralgia paresthetica: a case report and description of a new ultrasound-guided technique. Curr Sports Med Rep. 2011;10(2):99-104.

17. Onat SS, Ata AM, Ozcakar L. Ultrasound-guided diagnosis and treatment of meralgia paresthetica. Pain Physician. 2016;19(4):E667-669.

18. Patijn J, Mekhail N, Hayek S, Lataster A, van Kleef M, Van Zundert J. Meralgia paresthetica. Pain Pract. 2011;11(3):302-308.

19. Chhuttani PN, Chawla LS, Sharma TD. Meralgia paraesthetica. Acta Neurol Scand. 1966;42(4):483490.

20. Kitchen C, Simpson J. Meralgia paresthetica. A review of 67 patients.Acta Neurol Scand. 1972;48(5):547-555.

21. Rosencheck C. Meralgia paresthetica - its relation to osteoarthritis of the spinal vertebrae. JAMA. 1925;85(6):416-418.

22. Williams PH, Trzil KP. Management of meralgia paresthetica. J Neurosurg. 1991;74(1):76-80.

23. Elavarasi A, Goyal V, Singh MB, Padma Srivastava MV. Is triamcinolone an easy and efficient way to treat meralgia paresthetica? A cohort study. Ann Indian Acad Neurol. 2019;22(3):308-310.

24. Ducic I, Dellon AL, Taylor NS. Decompression of the lateral femoral cutaneous nerve in the treatment of meralgia paresthetica. J Reconstr Microsurg. 2006;22(2):113-118.

25. Dureja GP, Gulaya V, Jayalakshmi TS, Mandal P. Management of meralgia paresthetica: a multimodality regimen. Anesth Analg.1995;80(5):1060-1061.

26. Hui GK, Peng PW. Meralgia paresthetica: what an anesthesiologist needs to know. Reg Anesth Pain Med. 2011;36(2):156-161.

27. Khalil N, Nicotra A, Rakowicz W. Treatment for meralgia paraesthetica. Cochrane Database Syst Rev. 2008;3:CD004159.

28. Jackson TP, Gaeta R. Neurolytic blocks revisited. Curr Pain Headache Rep. 2008;12(1):7-13. 
29. Lee DG, Jang SH. Ultrasound guided alcohol neurolysis of musculocutaneous nerve to relieve elbow spasticity in hemiparetic stroke patients. NeuroRehabilitation. 2012;31(4):373-377.

30. Walker EL, Wright GK, Burnett CJ. Alcohol neurolysis of digital nerves. Proc (Bayl Univ Med Cent). 2016;29(3):331-332.

31. Hung JC, Azam N, Puttanniah V, Malhotra V, Gulati A. Neurolytic transversus abdominal plane block with alcohol for long-term malignancy related pain control. Pain Physician.2014;17(6):E755-760.

32. Fujita Y. CT-guided neurolytic splanchnic nerve block with alcohol.Pain. 1993;55(3):363-366.

33. Haim A, Pritsch T, Ben-Galim P, Dekel S. Meralgia paresthetica: a retrospective analysis of 79 patients evaluated and treated according to a standard algorithm. Acta Orthop. 2006;77(3):482-486.

34. Tumber PS, Bhatia A, Chan VW. Ultrasound-guided lateral femoral cutaneous nerve block for marelgia paresthetica. Anesth Analg.2008;106(3):1021-1022.

35. Kang J, Liu Y, Niu L, Wang M, Meng C, Zhou H. Anesthesia upstream of the alcoholic lesion point alleviates the pain of alcohol neurolysis for intercostal neuralgia: a prospective randomized clinical trial.Clinics (Sao Paulo). 2020;75:e1296.

36. Kocabas H, Salli A, Demir AH, Ozerbil OM. Comparison of phenol and alcohol neurolysis of tibial nerve motor branches to the gastrocnemius muscle for treatment of spastic foot after stroke: a randomized controlled pilot study. Eur J Phys Rehabil Med.2010;46(1):5-10.

37. Brinks A, Koes BW, Volkers AC, Verhaar JA, Bierma-Zeinstra SM. Adverse effects of extra-articular corticosteroid injections: a systematic review. BMC Muskuloskelet Disord. 2010;11:206.

38. Hollander JL. Intrasynovial corticosteroid therapy in arthritis.Md State Med J. 1970;19(3):62-66.

39. McCarty DJ Jr, Hogan JM. Inflammatory reaction after intrasynovial injection of microcrystalline adrenocorticosteroid esters. Arthritis Rheum. 1964;7:359-67.

40. MacMahon PJ, Eustace SJ, Kavanagh EC. Injectable corticosteroid and local anesthetic preparations: a review for radiologists. Radiology. 2009;252(3):647-661.

41. Akural E, Ojala RO, Järvimäki V, Kariniemi J, Tervonen OA, Blanco Sequeiros R. MR-guided neurolytic celiac plexus ablation: an evaluation of effect and injection sprerad pattern in cancer patients with celiac tumor infiltration. Cardiovasc Intervent Radiol.2013;36(2):472-478.

42. Kim BH, No MY, Han SJ, Park CH, Kim JH. Paraplegia following intercostal nerve neurolysis with alcohol and thoracic epidural injection in lung cancer patient. Korean J Pain.2015;28(2):148-152.

Table 1:Inclusion criteria of the study

1. Age [?]18 years

2. Pain or numbness on anterolateral thigh for [?] 6 months

3. Body mass index $<35 \mathrm{~kg} / \mathrm{m}^{2}$

4. American Society of Anesthesiologists (ASA) score grade I to III

5. Visual analog scale score for pain intensity $>5$ for participant's self-assessment of pain

6. No abdominal (gynecological or urological) surgery by Phannenstiel incision, open or laparoscopic inguinal herniorrhaphy or appendectomies, iliac bone graft harvest, pelvic osteotomies, vascular or orthopaedic operation to the pelvis, affected hip or affected extremity

7. Failure to respond to treament modalities, including physical therapy, nonsteroidal antiinflammatory drugs, pregabaline, gabapentin, and amitriptyline

Table 2:Exclusion criteria of the study

1. Pregnancy or lactation

2. Bilateral meralgia paresthetica

3. Body mass index $>35 \mathrm{~kg} / \mathrm{m}^{2}$

4. Previous surgery for meralgia paresthetica

5. Communication barriers and uncooperative cases

6. Any previous injection (local analgesic, corticostreoid, platelet-rich plasma, prolotherapy) to the lateral cutaneous femoral nerve

7. History of epilepsy, type 1 or 2 diabetes or hematologic disease 
8. Previous pelvis, hip or femur fracture

9. Arthritis of the pelvis, hip or knee

10. History of gout arthritis

11. History of systemic inflammatory autoimmune or peripheral vascular disease, such as deep venous thrombosis or bleeding disorders

12. Effusion around the injection site

13. History of lumbar disease (lumbar disc herniation, lumbar spinal stenosis, spondylolisthesis, spondylosis, spondyloarthritis) in magnetic resonance imaging

14. History of tendinopathy of the hip or pelvis

15. Pelvic or femoral bone tumor or cyst

16. Pelvic and retroperitoneal tumors

17. History of lumbar radiculopathy or piriformis syndrome

18. Leg length discrepancies

19. Scoliosis

20. Osteomyelitis or periostitis of the pelvis and affected extremity

21. Systemic infection

22. Tuberculosis infection

23. Joint, bone or skin infection in the affected extremity or pelvis

24. Trochanteric bursitis

25. Complex regional pain syndrome

26. Cardiac, liver or renal failure

27. Cardiac pacemaker

28. Abnormal erythrocyte sedimentation rate or C-reactive protein level

29. Known sensitivity or allergic reaction to bupivacaine

30. History of pelvis, hip or femur injury after meralgia paresthetica treatment had started through the 36-month follow-up point

\begin{tabular}{lll}
\hline Variable & Group 1 & Group 2 \\
\hline Gender (n) Male Female & 114 & 92 \\
Affected extremity Right Left & 105 & 65 \\
Age (y) Mean Range & $42.2 \pm 9.027-55$ & $40.8 \pm 7.3831-52$ \\
BMI (kg/m ${ }^{2}$ ) Mean Range & $29.0 \pm 2.224 .3-32.3$ & $30.0 \pm 3.322 .9-34.3$ \\
Symptom duration (mo) Mean Range & $12.4 \pm 1.111-14$ & $12.1 \pm 1.410-14$ \\
Follow-up period (mo) Mean Range & $28.7 \pm 2.624-33$ & $28.4 \pm 3.124-34$ \\
American Society of Anesthesiologists (ASA) score (n) Grade I Grade II & 96 & 74 \\
\hline
\end{tabular}

Table 3: Demographic patient data

\section{Hosted file}

Table 1.docx available at https://authorea.com/users/417637/articles/524653-effectivenessof-ultrasound-guided-corticosteroid-injection-and-alcohol-neurolysis-in-the-treatmentof-meralgia-paresthetica-during-a-28-month-follow-up-period-a-retrospective-cohort-study

\section{Hosted file}

Table 2.docx available at https://authorea.com/users/417637/articles/524653-effectivenessof-ultrasound-guided-corticosteroid-injection-and-alcohol-neurolysis-in-the-treatmentof-meralgia-paresthetica-during-a-28-month-follow-up-period-a-retrospective-cohort-study

\section{Hosted file}

Table 3.docx available at https://authorea.com/users/417637/articles/524653-effectiveness- 
of-ultrasound-guided-corticosteroid-injection-and-alcohol-neurolysis-in-the-treatmentof-meralgia-paresthetica-during-a-28-month-follow-up-period-a-retrospective-cohort-study 